\title{
PERFIL Y EVOLUCION DEL SISTEMA DE PARTIDOS EN CHILE
}

\author{
Paulo Hidalgo (*)
}

Chile, en comparación con otros países del continente, ha contado con un sólido sistema de partidos que históricamente fue el mediador privilegiado entre el Estado y la sociedad. Para comprender a cabalidad esta afirmación el paso obligado es situarse en las coordenadas de desarrollo de la sociedad chilena. En efecto, lo que en otros contextos latinoamericanos se verificó de manera asincrónica, en Chile se dio simultáneamente; es decir un proceso de industrialización sustitutiva (una industrialización "liviana" de bienes de consumo que antes se importaban) y la existencia por un largo período de un régimen político democrático con un proceso de democratización sustantiva que permitió unos grados de participación ampliados. Como lo han demostrado algunos notables historiadores políticos (1) la democratización chilena no fue un proceso unilineal y armónico sino que consistió en una incorporación gradual de diversos sectores sociales a la ciudadanía económica, social, cultural y política que se efectuaba en forma mediatizada, segmentada, conflictiva - con marchas y retrocesos- en función de la efectiva capacidad de organización y presión sobre el Estado que a la postre se erigía en el único referente de la acción colectiva.

En esta trama el sistema de partidos y la clase política que le era consustancial, configuraban el instrumento privilegiado para acceder al Estado y el único puente para que los diversos sectores sociales se tornaran en actores de relevancia nacional. Así la singularidad política del país consistía en el entrelazamiento, solapamiento o imbricación que se verificaba entre élite política — partido y grupo social (2).
Este fenómeno con variantes y matices importantes es generalizable a América Latina en su conjunto como bien lo ha destacado recientemente Ludolfo Paramio y contribuyó a la larga a una marcada sobrecarga del Estado. Según argumentos de Marcelo Cavarozzi la ausencia en América Latina de una cultura política centrada en una legitimidad estatal de tipo legal racional o si se quiere apegada a los procedimientos de selección de gobernantes y toma de decisiones, condujo a la configuración de un Estado doblemente cautivo y genéticamente débil: "el Estado depende en su legitimidad de su capacidad para ofrecer mejoras económicas a un conjunto de actores sociales que, a su vez, dependen del Estado sustancialmente para mantener su posición económica y social" (3).

Volviendo al caso chileno es posible destacar ciertos rasgos positivos de esta particular estructura política. En primer lugar su notable estabilidad. Sin abundar en mayores detalles la longevidad del sistema político chileno se explica en gran medida por una temprana - según canones latinoamericanos- formación del Estado que se asienta ya en 1830 sobre la base de la hegemonía de la oligarquía agraria del valle central. Además el ente estatal cumple un papel clave en la canalización y distribución de los recursos económicos. A él se irán integrando otras facciones oligárquicas, tanto como sectores medios - el Partido Radical- y expresiones del artesanado y del embrionario movimiento popular —el Partido Demócrata-. Más tarde en la década del treinta se incorporarán al Estado socialis-

(*) Chileno. Licenciado en Sociología y Ciencias Políticas por la Universidad de York, Inglaterra. Master en Ciencias Sociales por FLACSO, México. Actualmente es investigador visitante en la Fundación Pablo Iglesias, Madrid.

(1) Mario Gongora, Ensayo histórico sobre la noción de Estado en Chile en los siglos XIX y XX. Ed. Universitaria, 1986, Chile; Sergio Villalobos, (comps), Historia de Chile. Ed. Universitaria, 1990, Chile.

(2) Manuel Antonio Garretón, El proceso político chileno. Flacso, Chile, 1983; Arturo Valenzuela, The breakdown of democratic regimesChile. Johns Hopkins University Press, 1978, Estados Unidos.

(3) Ludolfo Paramio, El final de un ciclo y la crisis de unos actores: América Latina ante la década de los 90 . Ponencia presentada al XV Congreso Mundial de Ciencia Política. Buenos Aires 21-25 de julio de 1991. 
tas y comunistas, y más tarde la Democracia Cristiana. A esto hay que añadir el profundo arraigo originario de los partidos que no son meras entelequias de notables gestadas desde las alturas. Chile configura, como se sabe, formas de representación si se quiere marcadamente clasistas. La izquierda nace en la zona del puerto de Valparaíso y del norte minero, teniendo como caldo de cultivo la existencia de un proletariado que vive en condiciones de un capitalismo salvaje en comunidades cerradas que les hace generar un fuerte sentido de identidad y de oposición: el Partido Radical como una escisión temprana de corte jacobino de la oligarquía ilustrada y que más tarde nuclea a los sectores medios de origen estatal; el Partido Socialista que recoge a un amplio movimiento de capas medias, universitarios y trabajadores de la capital y otras ciudades importantes durante la crisis del 30 , etc. Es quizás solamente en la derecha en donde existe un cierto déficit de representación política en tanto siempre hubo una tensión irresuelta entre intereses corporativos de corto plazo y fallidas elaboraciones de proyectos socio-políticos de mayor envergadura (4).

En suma, la sociedad chilena a partir de la década del 30, en particular con el acceso al gobierno del Frente Popular, exhibe un alto grado de integración. Se trataba en definitiva del predominio de un sistema de partidos que presenta una oferta ideológica "completa". Es decir había una derecha, un centro y una izquierda lo que permitía una efectiva ubicación de la ciudadanía en algún punto del espectro. Aquí no sucedió el complejo fenómeno del populismo que como un movimiento político amorfo, heteróclito, transclase se agotaba en la energía y carisma de un gran caudillo nacional. La masa campesina era la gran excluida del arreglo político chileno y la derecha mantenía en el parlamento un gran poder de veto y tutela sobre este sector social a la vez que desarrollaba, como ya se dijo, una política de corte eminentemente defensivo.

\section{EL DERRUMBE DEL ARREGLO DEMOCRATICO}

Esta configuración política con el correr del tiempo mostró claros signos de desgaste. Se hicieron patentes algunas debilidades originarias como cambios en el campo y orientaciones de las fuerzas políticas que conducen finalmente al derrumbe de la democracia chilena. Esto merece algunas precisiones históricas.

El primer gran ciclo del sistema de partidos en Chile se cierra con la experiencia caudillista del general Carlos Ibáñez que se prolonga de 1952 a 1957. Él era un militar que había tenido presencia en la escena política durante la crisis salitrera (década del veinte) y la depresión del treinta como una de las figuras más descollantes junto a Marmaduke Grove (fundador entre otros líderes del Partido Socialista) de un estamento militar de oficiales jóvenes, descontentos con la estrechez de la sociedad oligárquica y en búsqueda de alternativas ante el vacío político de aquel entonces. En los cincuenta este líder que reemerge se propuso recoger cierto hastío social que estaba en fermentación por la actividad política canalizada exclusivamente por los partidos políticos que eran crecientemente percibidos como cliques estrechas (en particular se devalúa la imagen del Partido Radical que aparece carcomiendo y utilizando prebendariamente el aparato del Estado). Junto a éstos se hacen cada vez más claros los endémicos cuellos de botella del modelo económico que acusa signos de agotamiento. Ya a estas alturas el estancamiento de la agricultura como una suerte de bastión feudal de la clase terrateniente era insostenible. El énfasis en una política de sustitución de importaciones descuidó la posibilidad de ampliar y diversificar las exportaciones, generando de este modo un alto grado de estrangulamiento en la balanza de pagos. Únicamente el cobre cubría cerca del $70 \%$ de las remesas de divisas del país. Las presiones inflacionarias afectaron duramente el nivel de vida de la población; entre 1952-55 el ritmo de aumento anual de los precios al consumidor pasó del $12 \%$ al $86 \%$.

De este modo el tema principal de Ibáñez durante la campaña presidencial, ante este panorama que se describe, fue precisamente su ataque a los partidos (señalados como incapaces de representar a crecientes sectores sociales y de resolver los ingentes problemas económicos del país). Sin embargo, la gestión de Ibáñez que se presenta como un gran salvador nacional fracasa rotundamente. Políticamente es incapaz de articular "desde arriba", el desencanto con el sistema político histórico, como efectivamente ocurrió con los populismos clásicos de Perón o Getulio Vargas. Su gobierno se caracterizó por un marcado erratismo e ineficiencia que lo llevaron a aplicar indistintamente políticas de corte estatista, liberal, con un efímero plan recomendado por la famosa "misión klein sacks", que es desechado rápidamente por el empresariado a la luz de los "sacrificios" que requería orientaciones izquierdizantes y de derecha. En buenas cuentas Ibáñez no abordó coherentemente las limitaciones estructurales de la modernización incompleta de los gobiernos frente-populistas, tales como enfrentar el atraso agrario, la dependencia externa de un solo producto y el imperativo de una reforma económica global que desactivara los bloqueos económicos ya señalados, entre otros rezagos acumulados.

(4) Tomas Moulian, Desarrollo político y Estado de compromiso. Desajustes y crisis estatal en Chile. Estudios Cieplan N.. 8, 1982, Chile. 
Luego del "vendaval" ibañista reaparece el sistema partidario con mutaciones importantes (5). Se asiste a un paulatino debilitamiento del centro político representado en el Partido Radical cuya flexibilidad histórica hacia los dos polos del espectro partidario permitía el equilibrio del sistema político. El centro es ocupado progresivamente por la democracia cristiana que se caracteriza por un alto grado de rigidez y doctrinarismo que la lleva a esgrimir un proyecto político propio devaluando las alianzas políticas. Este conglomerado obtiene en 1945 un 2,57\% de los votos; en 1949 un 3,92\%. El gran repunte se produce en 1957 cuando logra un $9,94 \%$ de los votos y un $15 \%$ en Santiago - la capital- que consagra el liderazgo tanto partidario como nacional de Eduardo Frei que es elegido Senador. Luego en las elecciones presidenciales de 1964 Frei obtiene una de las votaciones más altas de la historia política del país, un $55,7 \%$ de la votación. Por otra parte, se consolida una izquierda con un creciente peso electoral: en 1958 obtiene un $28,51 \%$ de los votos y más tarde en 1964 un $37,7 \%$, cuando accede al gobierno con un $36,2 \%$. Estos resultados refuerzan en la izquierda la elaboración de una política que aspira a la formación de un "gobierno popular" desechando toda posibilidad de establecer alianzas políticas. En su versión más radicalizada la conquista del gobierno era percibida como un proceso ininterrumpido hacia la instauración del socialismo. Por último ante este cuadro de crecimiento de las alternativas reformistas y progresistas se genera un creciente anquilosamiento de la derecha que se encastilla aún más, a la vez de que opta por una política de "mal menor", apoyando en su momento sin condiciones (1964) a alternativas políticas como la Democracia Cristiana que bloquearan a toda costa un triunfo de la izquierda. La derecha se refunda en 1962 con la fusión de corrientes nacionalistas e históricas y comienza a elaborar una visión agresiva de la democracia, de los compromisos que se habían gestado en Chile, y gradualmente construye un proyecto político con claros signos autoritarios.

Como lo demuestra la descripción precedente el cuadro partidario que cristaliza en Chile presenta elementos evidentes de polarización. Si se pudiera recurrir a un término que grafique la situación es plausible aducir que a comienzo de los sesenta dejó de existir cualquier iniciativa estable y orgánica de cooperación política. El período de las denominadas "planificaciones globales" como lo acuñó un historiador clásico (6) comprendió el intento de desarrollar dos programas presidenciales que en verdad se proponían cambios ambiciosos y de largo alcance, sin las mayorías y el consenso político necesarios: el de Eduardo Frei entre 1964 y 1970 - la "Revolución en
Libertad" — y el de Salvador Allende - la "Vía Chilena al Socialismo"- entre 1970 y 1973. Estos signos de deterioro del sistema político, evidentes en una exacerbada partidización de la sociedad, un maximalismo ideológico flagrante y desencuentro sin retorno de las diversas fuerzas políticas fueron el telón de fondo del colapso. Se deben agregar los procesos vividos durante los tres agitados años de la Unidad Popular que terminaron de minar el terreno para cualquier acción política racional: la existencia de una movilización social extrema de carácter confrontacional, una marcada desarticulación de la sociedad-descomposición capitalista-, una fascistización y encono de las capas medias, y sabotaje abierto y desembozado de las clases empresariales, etc.

\section{LA TRANSICION A LA DEMOCRACIA: EL ESCENARIO, LA EMERGENCIA Y DESEMPEÑO DE LOS PARTIDOS}

En marzo de 1990 asume luego de un prolongado interregno militar el gobierno democrático de Patricio Alwyn, de filiación demócrata cristiana, encabezando una coalición de centro-izquierda básicamente junto al Partido Socialista (ya repuesto de sus luchas intestinas y reunificado en un solo tronco).

Es menester afirmar desde un comienzo que el pilar político fundamental sobre el cual descansa la transición política en Chile es el acuerdo sustantivo que se logra entre aquellos sectores políticos - ya mencionados - que se enfrentaron duramente durante prácticamente tres décadas: el centro político Demócrata-Cristiano y parte sustancial de la izquierda simbolizada en el Partido Socialista. Se debe agregar que aunque no participa en la coalición política, el Partido Comunista - aunque tardíamente y desgarrado por pugnas internas y mediando una radicalización de su juventud-, también se adhiere y llama a su militancia a votar por el candidato único de la oposición democrática.

Es casi un lugar común señalar en Chile que la transición fue en parte posible porque la oposición democrática elaboró un conjunto de pactos o entendimientos básicos para llevar al país a la democracia. $\mathrm{Si}$ se quiere esto se llevó a la práctica pagando determinados costos pero logrando beneficios indudables que han encaminado al país en una senda segura de gradual pero efectiva democratización. Tales pactos se pueden sintetizar esquemáticamente en cuatro puntos elementales: (7) (i) Pacto constitucional. Esta fue la certidumbre de la oposición luego de enconadas discusiones de que era impensable un cambio de

(5) Marcelo Cavarozzi, Manuel Antonio Garretón, (comps) Muerte y resurrección. Los partidos políticos en el autoritarismo y las transiciones del cono sur. Flacso, 1989, Chile; Norbert Lechner, El sistema de partidos en Chile. Una continuidad problemática. Documento de Trabajo N. 249, Flacso, 1985; Paulo Hidalgo, Pasado y presente de los partidos de izquierda. Un ensayo interpretativo. CED, 1985, Chile.

(6) Mario Gongora op cit... tercera parte.

(7) José Joaquín Brunner, Chile. Claves de una transición. Leviatan N.o 40. Verano 1990, España. 
régimen político sin reconocer la Constitución y la institucionalidad edificada por el régimen militar que se plasmaba en la Constitución de 1980. Esto llevo a la oposición a nuclearse primero en la denominada "Concertación por el NO". Es decir, la campaña masiva que desplegó la oposición primero para inscribir en los registros electorales a la mayor cantidad de votantes y luego a realizar una campaña para convocar a la ciudadanía a votar NO en el plebiscito llamado por el régimen militar para ratificar por otro período de ocho años el mandato del General Pinochet. Este evento electoral se realizó el 5 de octubre de 1988. La oposición democrática triunfó con un $56 \%$ de los sufragios y la opción "SI" (por la permanencia de Pinochet por ocho años) logró un $44 \%$ de votos. Este fue el acto desencadenante de la transición política. (ii) Pacto o acuerdo partidista. Esta fue la etapa de pasar de una coalición muy puntual e inestructurada a una coalición de corte más permanente con cierta normativa de funcionamiento y acuerdos más desagregados. Se fundó la Concertación de Partidos por la Democracia que postuló para la elección presidencial del 14 de diciembre de 1989 a Patricio Alwyn, como se adelantó al comienzo de este apartado. Este obtuvo un 55,17\% de los votos; el candidato del régimen militar Hernan Buchi obtuvo un $29,40 \%$ y Francisco Javier Errazuriz, un empresario exitoso que se lanza de improviso a la arena política con un discurso de corte populista, logra un $15,43 \%$ de los sufragios. (iii) Pacto electoral y de gobierno. Esto significó un complejo trabajo político de doble característica. Por una parte acordar la repartición de las diputaciones y senadurías en juego a través de una dilatada negociación (puesto que el sistema electoral que se heredaba del régimen militar forzaba un bi-partidismo y por ende a crear dos grandes coaliciones para lograr captar la masa mayor de votos). Por otra parte, se elaboró por un amplio espectro de comisiones profesionales y técnicas que fueron un verdadero gabinete en la sombra, un programa de gobierno que contemplaba medidas destinadas a democratizar plenamente al país (democratizar la gestión municipal), buscar resolver de la mejor manera la terrible herencia de la violación a los derechos humanos y buscar aliviar mediante ciertas reformas (laboral y tributaria) los acuciantes problemas sociales de falta de servicios mínimos, salarios deprimidos y desempleo. Se debe consignar que entre el plebiscito y la elección presidencial se acordaron entre el gobierno militar saliente y la oposición democrática triunfante una serie de reformas a la constitución votadas en un referéndum ad-hoc que a lo menos amortiguó los aspectos más autoritarios que ella aún presenta: permanencia del general Pinochet en la Comandancia en Jefe del Ejército, la existencia de senadores designados, la inamovilidad de la mayoría de los alcaldes del país, la existencia del
Consejo de Seguridad Nacional. (iv) Pacto en torno a las claves del desarrollo del país. Se refiere a ciertos acuerdos de más largo plazo en torno al imperativo de la mantención de una economía abierta, con una fuerte inversión privada, tanto nacional y extranjera, y a reconocer el papel central del mercado -en consonancia con una readecuación del rol del estado- como asignador de recursos. En suma desde un comienzo se aplacó o desactivó ante los inveterados temores de la clase empresarial nacional cualquier amenaza al funcionamiento del régimen de acumulación capitalista en el país.

Como se ha demostrado en estudios de sistema de partidos que se mantuvieron "congelados" por un período prolongado bajo condiciones autoritarias, los grados de continuidad de las lealtades políticas y la capacidad de mantención en algunos casos de una mínima y sólo simbólica estructura partidaria avalan la afirmación del profundo arraigo que alcanzan - a nivel de mentalidades y percepciones profundas en torno a la política - identidades políticas determinadas. A este respecto Chile no ha sido la excepción. A pesar del particular encono desplegado para "borrar" del mapa político tanto a los partidos de izquierda como de centro ellos lograron perdurar y más aún reproducirse vicariamente en condiciones en extremo hostiles. Esto se puede apreciar aunque sea en forma muy somera describiendo el desempeño electoral del arco político del país en las elecciones parlamentarias, tomando en consideración que la ley electoral premia a los partidos de mayor envergadura. El efecto directo de esta legislación fue que la oposición democrática tuvo que pactar con gran detalle y establecer una mutua cesión de escaños parlamentarios para maximizar la votación, lo cual por cierto no hizo reflejar a cabalidad las identidades y plena capacidad de representación de cada partido. La derecha se presentó aliada en sus dos componentes más importantes. La Unión Demócrata Independiente, más apegada al régimen militar y a la figura de Pinochet, y Renovación Nacional, de corte más modernizante y preocupada de "lavarse el rostro" y mostrar credenciales democráticas. Configuraron la coalición Democracia y Progreso. Lograron un $33,35 \%$ en la elección de diputados y un $35,4 \%$ en la elección de senadores. En general la derecha tiene como límite probable entre un cuarto y un tercio del electorado.

El centro político; es decir la Democracia Cristiana que supera su tendencia histórica de "camino propio" (imponer su propio proyecto político y mostrarse renuente a pactar con otras fuerzas políticas) y opta por una alianza perdurable con un sector clave de la izquierda obtiene la primera mayoría relativa en las parlamentarias. En diputados obtiene un $26,14 \%$ de los votos y se empina a un $32 \%$ de senadores. Una parte de la izquierda se presenta —debido a las restricciones legales aún vigentescomo Partido por la Democracia, cuyo eje central es 
un sector del Partido Socialista que realiza una profunda crítica de su quehacer pasado y emprende una renovación ideológica global y se compromete firmemente con el régimen democrático. Otro sector de la izquierda, básicamente el Partido Comunista y un sector ortodoxo del Partido Socialista que aún permanece en la matriz clásica marxista, se presentan a la disputa electoral como partido Amplio de Izquierda Socialista. Este último conglomerado que se congrega bajo el rótulo de Unidad para la Democracia obtiene un $5,31 \%$ de votos en diputados y un $4,24 \%$ de votos para senadores. Por otra parte, el Partido Por la Democracia que se integra a la concertación de partidos por la democracia logra el 10,98\% en la votación para diputados y el 11,9\% de la votación para senadores. Aunque muy por debajo de la Democracia Cristiana este partido se convierte en el segundo partido de la concertación y en el tercero en representación parlamentaria en el país. Una vez que el Partido Socialista se reunifica luego de las elecciones, se consolida como segunda fuerza política en la coalición de gobierno obteniendo seis ministerios del primer gabinete. (Ver cuadros N. $1,2,3$ ).

Muy gruesamente se puede concluir este apartado señalando que el sistema partidario mantiene sus lealtades y referentes clásicos pero más bien con una tendencia a la constitución, como acertadamente lo ha señalado Manuel Antonio Garretón, de un entramado partidario de cuatro polos en vez de los históricos enfrentados tres tercios. (8) Tal aseveración se sintetiza en los siguientes aspectos: (i) un primer polo constituido por una derecha escindida; un sector de ella heredera del autoritarismo e incluso de la figura de Pinochet versus una derecha que busca modernizarse afanosamente y declara una firme adhesión al régimen democrático. (ii) un segundo polo representado por la Democracia Cristiana que pendula entre un retorno a su viejo hábito de "camino propio" o decidirse sin ambigüedades a establecer una alianza de largo plazo con la izquierda socialista. (iii) un tercer polo expresado en el Partido Socialista ya unificado cuyo desafío es convertirse en una fuerza política plenamente moderna, programática y autónoma del centro político y de la izquierda comunista. (iv) por último se encuentra la izquierda comunista. Su disyuntiva es si enarbola definitivamente una política radical protestataria de pura reivindicación y expresividad ética o se subordina a la hegemonía de la izquierda socialista.

\section{ALGUNOS TEMAS DE FONDO}

En general se puede sostener, como se percibe del análisis precedente, que los partidos políticos chilenos ya hicieron su "camino de damasco" en recono- cer los errores que cometieron en el pasado y sin duda que han enmendado rumbos de manera significativa. Sin embargo, los procesos políticos son lentos y es posible a lo menos reseñar el tipo de desafíos que enfrenta el esquema partidario hacia adelante. A mi juicio lo fundamental es la capacidad de apertura que éste posea para adoptar lo que se pudiera rotular como una radicalidad democrática. Recrear una sociedad plenamente participativa y aportar a la creación de un sistema político sólido se ciernen como los desafíos de este fin de siglo y en imperativo para los conglomerados partidarios.

Por una parte, los partidos deben encarar una profunda modernización de sus estructuras y funcionamiento generando canales de comunicación fluidos y sistemáticos hacia la sociedad civil, y deben aprender a transitar y no confundir la especificidad de los tres ámbitos en que se desenvolverán: el Estado, el Parlamento y las organizaciones intermedias de la sociedad civil.

Esta certeza supone, a mi juicio, en particular para una alternativa progresista, la paulatina elaboración de un proyecto político que ponga en el centro del debate público la compleja temática del tipo de proyecto nacional en sentido lato que nos acercaría a una forma de convivencia más libre e igualitaria. Aquí es especialmente relevante el espectro de reformas que es dable plantear al conjunto de las fuerzas políticas nacionales que sean capaces de sincronizar la competitividad, el crecimiento económico, la productividad con el avance del poder de la sociedad - los trabajadores- sobre las élites y el Estado. Lo recién señalado puede actuar como un mecanismo evitatorio de una política simplemente pragmática, conservadora y acomodaticia. La condición política sine-quanon de esta demanda es la configuración a largo plazo en el país de un bloque de centro e izquierda que admitiendo grados de competencia y diferenciación en su interior compartan un gran programa mayoritario por los cambios sociales.

Por otra parte, contrarrestar una tendencia a la oligarquización de la política - aspecto de cierta vigencia en el panorama político actual- y a un creciente "autismo" — con nexos poco claros hacia la sociedad - de la razón técnica nos lleva a enfatizar el tipo de recursos que la democracia puede desarrollar. Entre ellos, se encuentra el robustecimiento de las instancias de participación de base e intermedias - la grass roots democracy de la tradición sajona-; el despliegue de una política periódica de rendición de cuentas del representante - cualquiera que él sea- ante sus representados - la accountability-. Una legislación que asegure una adecuada rotación de los cargos a todo nivel y una vitalización de la

(8) Manuel Antonio Garretón, Partidos políticos, transición y consolidación democrática. Revista Proposiciones. Enero 1990 Ed. SUR, Chile; Un análisis complementario se encuentra en la REIS, N. 50, abril-junio 1990, Rodrigo Baño, "Elecciones en Chile: ¿Otra vez lo mismo o al revés?" 
arena parlamentaria como espacio institucional de contrachequeo de la "performance" del gobierno en todos sus planos de actividad, etc.

Ahora que nos encontramos con bastante menos certidumbres que antaño y sin un modelo-teóricopráctico que nos ordene el mundo de una vez y para siempre, resulta más sano incorporar el conflicto y la discusión en torno a los límites de lo posible. Ya sabemos que más allá de nuestra voluntad se esconden formas de convivencia muy disímiles, como la barbarie del capitalismo salvaje que hemos vivido estos largos años, la inviabilidad y la anomia generalizada traducida en un lúcido cinismo, un colectivo social eficaz pero rutinizado gobernado por un estamento técnico unidimensional y totalizante, o la búsqueda ciega de satisfacción inmediata de los más variados grupos y sectores corporativos.

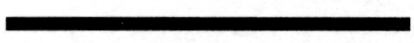

\section{APENDICE}

\section{Cuadro 1}

\section{RESULTADO ELECCION PRESIDENCIAL (Votos válidamente emitidos)}

Patricio Aylwin

$55,17 \%$

Hernán Büchi

$29,40 \%$

Francisco Javier Errazuriz

Cuadro 2

\section{ELECCIONES PARLAMENTARIAS. PORCENTAJE TOTAL DE VOTOS POR PACTO ELECTORAL}

\begin{tabular}{|c|c|c|}
\hline & Diputados & Senadores \\
\hline - Concertación por la Democracia.. & 51,48 & 54,63 \\
\hline — Unidad para la Democracia........... & 5,31 & 4,24 \\
\hline — Democracia y Progreso ................... & 34,18 & 34,85 \\
\hline - Otros pactos de derecha ................ & 9,03 & 6,28 \\
\hline TOTAL ................................. & 100,00 & 100,00 \\
\hline
\end{tabular}

Cuadro 3

PARLAMENTARIOS ELEGIDOS POR PARTIDO

\begin{tabular}{|c|c|c|}
\hline PARTIDOS & Diputados & Senadores \\
\hline - Democracia Cristiana ......................... & 39 & 13 \\
\hline — Renovación Nacional .................... & 34 & 11 \\
\hline — Partido por la Democracia............. & 17 & 6 \\
\hline 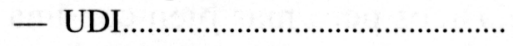 & 13 & 2 \\
\hline - Partido Radical .............................. & 5 & 1 \\
\hline — Partido Socialista (Almeyda) ........ & 6 & 1 \\
\hline — Izquierda Cristiana ........................ & 2 & \\
\hline - Centro Democrático Libre.............. & 1 & \\
\hline - Social Democracia ......................... & 1 & 1 \\
\hline — Partido Humanista ........................... & 1 & \\
\hline — Independientes ............................ & 1 & 3 \\
\hline — Designados .................................... & & 9 \\
\hline TOTAL..................................... & 120 & 47 \\
\hline
\end{tabular}

FUENTE: Situación Latinoamericana. CEOFAL - N. ํㅡ, Abril 1991, ESPAÑA.

\section{RESUMEN}

Este artículo analiza los aspectos principales del sistema de partidos chileno. En primer lugar, se estudian los aspectos principales de los partidos políticos y su configuración histórica. En segundo lugar, se ofrece una breve descripción del derrumbe de la democracia y el papel de los partidos en ese cuadro. En tercer lugar, se presenta un bosquejo de la transición a la democracia y luego se examina el desempeño de cada conglomerado partidario en las primeras elecciones fundacionales. Finalmente se esbozan algunos de los desafíos que se le presenta al sistema partidario desde un punto de vista que enfatiza la noción de una democracia radical.

\section{ABSTRACT}

This article discusses the main aspects of the chilean party system. Firstly there is a brief analysis of the main features of the political parties and their historical configuration. Secondly there is a short description of the breakdown of democracy and the role of the parties in it. Thirdly there is an outline of the transition to democracy with the election of Patricio Alwyn and then we examine the performance of each party in the founding elections after the autocratic period. Finally we conclude with some of the challenges facing the new born chilean democracy, in particular with reference to a notion of a radical democracy. 